\title{
A Short Note on Compactness in Aligned Spaces and Higgs Spaces
}

\author{
J. M. S. Simões-Pereira \\ Departamento de Matemática, Universidade de Coimbra, Coimbra, Portugal, E-mail: siper@mat.uc.pt
}

Received: April 26, 2017 / Accepted: May 21, 2017 / Published: June 25, 2017

Abstract: We generalize to aligned spaces and to Higgs spaces the concept of compact topological spaces.

Key words: aligned spaces, Higgs spaces, compactness.

\section{Introduction}

Aligned spaces and Higgs spaces generalize topological spaces and, for this reason, I believe in their potential to play important roles. For the moment, however, they are not receiving as much attention as they deserve. In this note we extend to aligned spaces and to Higgs spaces the concept of compactness of topological spaces. Aligned spaces were introduced in [1], and dealt with in [2], [3] and [6], for example. As defined in [1], an aligned space, denoted $(V, C)$ is a set $V$ with a family $C$ of subsets, called convex sets, such that:

A1) $V, \varnothing \in C$; and

A2) $A, B \in C \Rightarrow A \cap B \in C$.

A topological space is an aligned space which satisfies the additional condition that unions of a finite number of sets of $C$ also belong to $C$.

Concerning Higgs spaces, denoted $(H, \delta)$, they appear in [4], [5], [6] and [7], for instance. To define them, $H$ is a set and $\delta$ is the so-called derived operator which maps the set $P(H)$ of all subsets of $H$ into itself and which satisfies the following two conditions:

Corresponding author: J. M. S. Simões-Pereira, Departamento de Matemática, Universidade de Coimbra, Coimbra, Portugal, E-mail: siper@mat.uc.pt.
B1) For $A, B \subseteq H \quad$, if $A \subseteq B$ then $\delta(A) \subseteq \delta(B)$; and

B2) If $x \in \delta(A)$ then $x \in \delta(A-\{x\})$.

A Higgs space becomes a topological space when the following additional conditions are satisfied:

B3) $\delta(\varnothing)=\varnothing$; and

B4) $\delta(A \cup B) \subseteq \delta(A) \cup \delta(B)$. Since, by $\mathrm{B} 1$, $\delta(A) \cup \delta(B) \subseteq \delta(A \cup B)$, here, in B4, we can write $\delta(A \cup B)=\delta(A) \cup \delta(B)$.

Now, given a Higgs space, call a set $A \subseteq H$ closed when $\delta(A) \subseteq A$ or, equivalently, $A=A \cup \delta(A)$. Trivially, when $A$ is closed, we have

$$
\delta(A) \equiv \delta(A \cup \delta(A)) \subseteq A \equiv A \cup \delta(A)
$$

For any $A \subseteq H$, we call $A \cup \delta(A)$ the closure of $A$. The reason is that $A \cup \delta(A)$ is the smallest closed set containing $A$. In fact, let $A \subseteq B$ and $B$ closed. We may write $\delta(A) \subseteq \delta(B) \subseteq B$. From these inequalities we get $A \cup \delta(A) \subseteq B \cup B=B$, hence $B$ cannot be smaller than $A \cup \delta(A)$.

If we write $F(A)=A \cup \delta(A)$, it is easy to verify that $F$ is a closure. In fact,

C1) $A \subseteq F(A)$;

C2) $A \subseteq B \Rightarrow F(A) \subseteq F(B)$; and

C3) $F^{2}(A)=F(A)$. This follows from the fact that $\delta(A \cup \delta(A)) \subseteq A \cup \delta(A)$. 
If the derived operator meets conditions $\mathrm{B} 3$ and $\mathrm{B} 4$, then we have:

C4) $F(\varnothing)=\varnothing \cup \delta(\varnothing)=\varnothing$; and

C5) $F(A) \cup F(B)=A \cup \delta(A) \cup B \cup \delta(B)$ $=A \cup B \cup \delta(A \cup B)=F(A \cup B)$

The closure we have just defined with these five axioms is a topological closure, hence we have here a topological space defined in terms of a closure operator.

In a Higgs space, given two complementary sets $A$ and $A^{c}$, if one of them is closed we say that the other one is open. A set $A$ is open, if and only if $A \cap \delta\left(A^{c}\right)=\varnothing$. In fact, with $A \cap A^{c}=\varnothing$ and $A \cup A^{c}=H$, if $A^{c}$ is closed, that means $\delta\left(A^{c}\right) \subseteq A^{c}$, then $A \cap \delta\left(A^{c}\right)=\varnothing$. On the other hand, suppose now that $A \cap \delta\left(A^{c}\right)=\varnothing$. Since $A \cap A^{c}=\varnothing \quad$ and $\quad A \cup A^{c}=H \quad$, we get $\delta\left(A^{c}\right) \subseteq A^{c}$ which means $A^{c}$ is closed and, by the very definition, $A$ is open.

When dealing with an aligned space $(V, C)$, let $Q$ be the family of the complements of its convex sets. Call them concave sets. In terms of concave sets, we may present an alternative definition:

Let $V$ be a set and $Q$ a family of subsets of $V$ such that:

D1) $\varnothing, V \in Q$;

D2) $A, B \in Q \Rightarrow A \cup B \in Q$.

Then $(V, Q)$ is an aligned space and the sets of the family $Q$ are called its concave sets.

If the finite intersections of sets of $Q$ are also in $Q$, then $(V, Q)$ is a topological space whose open sets are the sets of $Q$.

\section{Aligned Spaces}

As pointed out in [6], many basic concepts in the study of topological spaces may be extended to aligned spaces. Let us look here at the concept of compact space. Let $(V, Q)$ be an aligned space defined in terms of concave sets. We have:
Definition of a compact aligned space: The aligned space $(V, Q)$ is said to be compact when every covering of $V$ by concave sets has a finite sub-covering.

To give a definition in terms of convex sets, let us first recall that a family $F$ of sets is said to have the finite intersection property when every finite subfamily of $F$ has a non-empty intersection. Note that a family with the finite intersection property may have an empty intersection: an example is the family of the open intervals $\{(0,1 / n) \mid n=1,2,3, \ldots\}$. We prove:

Theorem 1: The aligned space $(V, C)$ is compact if and only if every family of convex sets with the finite intersection property has itself a non-empty intersection.

Proof: 1. Suppose $V$ is a compact aligned space and $C$ is a family of convex sets such that each finite subfamily $\left\{C_{1}, \ldots, C_{n}\right\}$ has a non-empty intersection; this means, $\exists a \in C_{1} \cap \ldots \cap C_{n}$. Their complements are concave sets $H_{1}, \ldots, H_{n}$ and $a \notin H_{1} \cup \ldots \cup H_{n}$. In words: if, for every finite family of convex sets, there exists one point $a$ which belongs to their intersection, then, for every finite family of concave sets, there is one point which does not belong to their union.

As a consequence, given a covering $H$ of $V$ by concave sets, we cannot extract from it a finite sub-covering, which contradicts the hypothesis that $V$ is compact. If $V$ is in fact compact, then $H$ is not a covering, there will be at least one point, say $a$, not covered by any set in $H$; therefore, $a$ belongs to all sets in $C$, that means, $C$ has a non-empty intersection.

2. Now suppose $V$ is not compact, that means, there is a covering $H$ by concave sets such that no finite subfamily of $H$ covers $V$; by other words, given a finite subfamily $H_{1}, \ldots, H_{n}$ there is at least one point $a \notin H_{1} \cup \ldots \cup H_{n}$. This is true for all 
finite subfamilies of $H$. Let $C$ be the family of the complementary sets of the sets in $H$. There is always one point, say $a$, such that $a \in C_{1} \cap \ldots \cap C_{n}$. But since $H$ covers $V$, there is no point $a \notin H$, hence there is no point which belongs to all sets in $C$; hence the intersection of all sets of $C$ is empty, although $C$ has the finite intersection property. This completes the proof.

Trivially, we can read the statement of Theorem 1 as a second definition of a compact aligned space, given in terms of convex sets.

\section{Higgs Spaces}

Since we first defined Higgs spaces in terms of closed sets, let us give a first definition of compactness as follows:

Definition of a compact Higgs space: The Higgs space $(H, \delta)$ is said to be compact when every family of closed sets with the finite intersection property has itself a non-empty intersection.

Traditionally, compactness of topological spaces is defined in terms of open sets. We may honour this tradition by proving now the following result:

Theorem 2: A Higgs space $(H, \delta)$ is compact (according to the above definition) if and only if every covering of $H$ by open sets has a finite sub-covering.

Obviously, to prove this theorem is the same as to prove that, in a Higgs space, the following two statements are equivalent:

Statement X: Every family of closed sets with the finite intersection property has itself a non-empty intersection.

Statement Y: Every covering of $H$ by open sets has a finite sub-covering.

There is no need to explicit the details of such a proof. We just ask the reader to follow the proof of Theorem 1, replacing convex sets by closed sets and concave sets by open sets.

\section{References}

[1] Paul H. Edelman: Meet-distributive lattices and the anti-exchange closure, Algebra Universalis 10 (1980), 290-299.

[2] Martin Farber and Robert E. Jamison: Convexity in graphs and hipergraphs, SIAM Journal on Algebraic and Discrete Methods 7 (1986), 433-444.

[3] Paul H. Edelman and Robert E. Jamison: The theory of convex geometries, Geometriae Dedicata 19-3 (December 1985), 247-270.

[4] D. A. Higgs: Matroids and duality, Colloquium Mathematicum 20 (1969) 215-220.

[5] D. A. Higgs: Infinite graphs and matroids, Recent Progress in Combinatorics (ed. W. T. Tutte), Academic Press, New York and London (1969), 245-253.

[6] J. M. S. Simões-Pereira: Alignments, topologies, convex geometries and Higgs spaces, Journal of Mathematics and System Science 4 (2014), 158-163.

[7] J. M. S. Simões-Pereira: Matroids, graphs and topology, in: Proc. 5th Conf. Combinatorics, Graph Theory, and Computing, in Boca Raton, Florida, Feb. 25 - March 1, 1974; Congressus Numeratium X (Utilitas Mathematica Publishing Inc., Winnipeg, Canada, 1974), 145-155. 